\title{
aichinger - digital. Digitale Corpusanalyse in der Literaturwissenschaft am Beispiel von :aichinger
}

\author{
Marlene Csillag \\ and Mathias Müller \\ ACADEMIAE CORPORA \\ (ÖAW)
}

\author{
Andreas Dittrich \\ Fellow, DLA Marbach / \\ ACADEMIAE CORPORA \\ (ÖAW)
}

\author{
Katharina Godler \\ Robert Musil Institut \\ für Literaturforschung / \\ Kärntner Literaturarchiv
}

\begin{abstract}
Vorgestellt wird ein Projekt, das Methoden digital gestützter Corpusanalyse in der Literaturwissenschaft paradigmatisch zu entwickeln sucht. Als Untersuchungsbeispiel wurde das Werk der österreichischen Autorin Ilse Aichinger (1921-2016) gewählt. Der primäre Fokus der Untersuchung liegt auf der literarischen Repräsentation von Raum. Bisherige Untersuchungen ihres Werks haben erwiesen, dass Aichingers Bezugnahmen auf Orte und Ereignisse in Wien zentrale Bedeutung zukommt (Fässler, 2011). Ziel des Projekts ist es, grundlegende Strukturen in Aichingers Referenzierung auf Orte zu ermitteln und deren Zusammenhang zur historischen Erfahrung herauszuarbeiten, deren Darstellung im Zentrum ihres Werks steht.
\end{abstract}

\section{Aichinger ver suchen}

Innerhalb des Projektes :aichinger (in Worten: DOPPELPUNKT AICHINGER), beschäftigt sich eine Gruppe junger Forscherinnen und Forscher seit mehreren Jahren mit dem Werk der österreichischen Autorin Ilse Aichinger. Ausgehend von einer Lehrveranstaltung an der Universität Wien entstand 2015 mit dem damaligen ICLTT (in Worten: INSTITUT FÜR CORPUSLINGUISTIK UND TEXTTECHNOLOGIE), heute AC (in Worten: ACADEMIAE CORPORA), eine Kooperation an der Österreichischen Akademie der Wissenschaften.

Das Aichinger-Corpus umfasst das digitalisierte und annotierte Werk Ilse Aichingers. Für die Erstellung des Corpus wurde die beim S. Fischer Verlag publizierte Werkausgabe als Ausgangspunkt gewählt. Zusätzlich wurden die in den 2000er Jahren in den Verlagen S. Fischer und Edition Korrespondenzen veröffentlichten Texte, die nicht in der Werkausgabe enthalten sind, berücksichtigt.

Das Corpus enthält 380 Texte und 379,914 word-tokens. Die Texte wurden Part of Speech (PoS) getaggt, sowie alle Ortsnennungen mit einem rs-tag versehen und anschließend in eine CQP-Umgebung (Corpus Query Processor) eingebunden. Desweitern sind in den Metadaten die Entstehungszeit, die Erstpublikation und die Erstbuchpublikation berücksichtigt, soweit diese Informationen eruierbar waren.

Die literaturwissenschaftliche Arbeit mit digitalen Corpora beginnt mit dem Text. Aichingers erste selbstständige Publikation und ihr einziger Roman trägt den Titel Die größere Hoffnung. Eine einfache Suche in dem Aichinger-Corpus nach dem Lemma „Hoffnung“ ergibt dabei, dass das Wort „Hoffnung“ 95 mal in 44 verschiedenen Texten vorkommt. 86 mal in der Form „Hoffnung“; 9 mal in der Form „Hoffnungen“. Am häufigsten treffen die Lesenden auf das Wort „Hoffnung“ in ihrem Debütwerk Die größere Hoffnung, nämlich 17 mal. Und es zeigt sich auch, dass Hoffnung ein Wort ist, das sich durch das gesamte Werk von Ilse Aichinger zieht. In fast jedem Text, das sie veröffentlicht hat, und beinahe jedem Band der Werkausgabe trifft man auf „Hoffnung“ in verschiedensten Wortformen.

Der zweite Band der Werkausgabe Der Gefesselte hat uns zur Suche nach „Fesseln“ in allen Variationen veranlasst, zum Beispiel, als „Fessel“, ,gefesselt“, „Fesselung“. Erzielt haben wir dabei 98 Treffer, aber nur in vier verschiedenen Texten. Die meisten Fundorte des Wortes befinden sich in der Erzählung „Der Gefesselte“ des gleichnamigen Erzählbandes. Ein amüsanter Fund dabei: In dem Text „Hilfsstelle“ aus Kleist, Moos, Fasane gibt es einen Fesselballon.

Diese Art der einfachen Suche bzw. das Durchsuchen nach einem bestimmten Begriff, lässt sich natürlich unendlich fortsetzen. Ein weiteres Beispiel: Im ersten Kapitel von Die größere Hoffnung trifft 
Ellen in ihrer Fingerreise entlang einer Landkarte auf einen Haifisch für den, ebenso wie für die Kinder jüdischer Eltern, niemand bürgen wollte. Bei der Suche nach „Hai“ findet sich neben den „Haien“, den „Haifischen“, den „Haifischnetzen“ und den „Hainen” auch „Jörg Haider“: „Den augenblicklichen Zustand der FPÖ definiert Jörg Haider als »schwach, krankhaft und in der Existenz gefährdet «." (Aichinger, 2006, 43)

Natürlich kann die Abfrage auch komplexer gestaltet werden: Interessante Suchmöglichkeiten ergeben sich mit der Silbe „ver“, einer Silbe, die im Werk Aichingers sehr präsent ist und von ihr in einem Interview, als eine „Verschwinden-Silbe“ (Aichinger, 2011, 35) bezeichnet wird. In dem Text „Schnee“ schreibt sie davon, dass „,ver“ „,auf got. fra zurück[geht], so, wenn der Sinn eines Verschwindens oder Zugrundegehens vorliegt [... ]" (Aichinger, 1991c, 114).

In welcher Form „ver“ im Werk Aichingers aufscheint, kann durch das Corpus schnell ermittelt werden. Eine einfache Suche nach „ver“ als Vorsilbe ergibt 3839 Treffer in 301 Texten; darunter unterschiedliche Wörter und Wortarten wie „Vermessenheit“, „Vertrauen“, „versteckt“, „,verschwunden“, „,verstummen“.

„Ver" kommt nicht nur als Vorsilbe zum Vorschein sondern auch als Endung, wenn auch weniger prominent. 102 Treffer in 30 Texten, darunter Namen wie „Dover“, „Xaver“ oder Nomen wie „Manöver“, „Pulver“, „Revolver“.

Eine weitere Ebene der Suche ergibt sich über die PoS-Tags, nach denen ebenfalls gesucht werden kann. Diese ermöglichen auch weitere Fragestellungen und Forschungsmöglichkeiten. Beispielsweise können ohne größere Probleme alle Zahlen im Corpus gefunden werden, in dem einfach nach dem STTSTagset „CARD“ gesucht wird. Dabei werden gleichsam die in Buchstaben ausgeschriebenen Zahlen, wie auch numerische Ziffern gefunden. Es können Listen mit allen Substantiven, allen Verben, Adverbien, adverbialen Interrogativpronomen oder auch einfachen Nichtworten (Sonderzeichen, Kürzel) erstellt werden. Am interessantesten erscheint es aber, wenn man die Suche nach PoS-Tags mit der Suche nach Wörtern oder Lemmata kombiniert.

Man kann also - zurückkommend auf den Roman Die größere Hoffnung - nicht nur jene Stellen finden, an denen Ellen, die Protagonistin des Textes, genannt wird, sondern auch, was sie gerade tut, indem z.B. nach „Ellen“ und dem nachfolgenden Verb ( [ lemma="Ellen"] [pos="V • *"]) gesucht wird. So wird ersichtlich, dass „Ellen schob“, „Ellen fror“, „Ellen schrie“, „Ellen weinte“, „Ellen wühlte“ und mehr.

Weitere Abfragemöglichkeiten mit den PoS-Tags beinhalten die Suche nach Nomen ( [pos="NN"]): „Kap“, „Hoffnung“, „Meer“, „Schiffahrtslinien [sic!]“; die Suche nach zwei Nomen verbunden durch eine Konjunktion $([p \circ s=" N N "][p o s=" K O N "][p o s=" N N "]$ : „Blumen und Vögel“, „Sonne und Mond“, „Tage und Nächte“, „Qual und Bitterkeit“. Oder, evoziert durch den Komparativ im Titel Die größere Hoffnung, welche Wörter, vor allem welche Substantive, mit „größer“ adjektiviert sind: „größerer Bursche“, ,größere Entdeckerin“, ,größere Hoffnung“, ,größeres Wagnis“.

Um diese Ergebnisse zu präzisieren bietet CQP ein weiteres Tool. Die Suchergebnisse können, z.B. der Häufigkeit nach, geordnet werden. Am vorangegangenen Beispiel ,größer“ + Substantiv folgen auf die häufigste Nennung „größere Abenteuer“ die Treffer „größere Gesellschaft“, „größere Hoffnung“, „größere Vorsicht".

Werden beispielsweise die Treffer, die mit der Vorsilbe „ver“ beginnen, nach der häufigsten Verwendung angeordnet, ist ersichtlich, dass viele der Ergebnisse Verben sind, die mit dem Verschwinden assoziiert werden können: „,vergessen“, „,verloren“, „,verlassen“.

Die Wörter, die auf ,ver“ enden, haben keine derartige Gemeinsamkeit.

„Dover“ aber, das mit 38 Treffern deutlich vor den zehn Treffern von „Manöver“ liegt, hat, wie die Vorsilbe „ver“, einen merklichen Bezug zu vielen Texten von Ilse Aichinger. „Eine Fliege kroch von Dover nach Calais“ (Aichinger, 1991a, 9), jenen Küstenort der von Helga Michie (geborene Aichinger), der Schwester von Ilse Aichinger auf ihrer Reise ins Londoner Exil passiert wurde. Und von Dover aus kann die Welt aus den Angeln gehoben werden (Aichinger, 1991d, 41).

Eine weitere Funktion des CQP ist es, die Suchergebnisse nach den Texten zu ordnen in denen sie vorkommen. Die bereits erwähnten „Fesseln“ kommen in unterschiedlichsten Variationen, am häufigsten aber in „Der Gefesselte“, vor, und zwar 89 mal, gefolgt von Die größere Hoffnung mit drei mal. „Hoffnung“ 
wiederum kommt im gerade genannten Roman am häufigtsten v or, g efolgt v on d en Texten „Nach der weißen Rose“ und „Rede an die Jugend“.

Diese letzte Funktion des CQP ordnet die Texte aber nicht nur nach der Anzahl der Treffer in der Suche, sondern vor allem über das Verhältnis von Treffern und Textlänge, ausgedrückt in "Frequency per million words”. Dadurch ändert sich natürlich auch das Suchergebnis.

Das Wort „Hoffnung“ kommt im Verhältnis zur Textlänge am häufigsten in dem Text „In den verschwundenen Schnee“ vor (Aichinger, 2005, 81-84). Am zweithäufigsten werden die Aufzeichnungen zum Jahr 1959 aus dem Erzählband Kleist, Moos, Fasane angezeigt. Zwei Problemfelder werden innerhalb des Aichinger-Corpus sichtbar:

1. Es ist recht klein für ein Corpus. Das bedeutet: Viele der quantitativen Ergebnisse sind nicht besonders repräsentativ. In dem oben genannten Beispiel fällt Die größere Hoffnung, da es sich um den längsten Text handelt, sogar aus dem Ergebnis heraus.

2. Das publizierte Werk von Ilse Aichinger, wie es in dem Corpus zu finden ist, ist sehr uneinheitlich. Es besteht aus einem Roman, mehreren längeren und teils sehr kurzen Erzählungen, einem Gedichtband, einem Hörspielband, einem Band mit Szenen und Dialogen sowie weiteren Bänden mit Texten, die ursprünglich in Zeitungen erschienen sind und die nicht einfach einem Genre zuzuordnen sind.

Gerade die Kombination dieser beider Problemfelder führt zu statistischen Verfälschungen.

Zumindest der zweite Punkt lässt sich durch die Erstellung verschiedener Subcorpora etwas minimieren. Um repräsentativere Ergebnisse zu erzielen erscheint es sinnvoll in den Abfragen die dramatischen bzw. szenischen oder die poetischen Texte von der Prosa trennen. So könnte man auch Subcorpora erstellen, die alle Prosatexte enthalten, ohne den kurzen Aufzeichnungen aus den Notizbüchern von 1950-1985 bzw. den Kurzschlüssen, die wohl eher als Prosagedichte zu lesen sind. Auf diese Art und Weise können statistisch relevantere Ergebnisse erzielt werden. Allerdings ist diese Methode nur bei manchen Abfragen anwendbar und nicht für jede Forschungsfrage 'geaichnet'.

Dazu muss aber gesagt werden, dass die literaturwissenschaftliche Arbeit mit einem Textcorpus nicht immer von statistischen und vor allem nicht ausschließlich von quantifizierbaren Methoden abhängig ist. Ein Beispiel für einen Fund, der mit klassischen literaturwissenschaftlichen Methoden schwer auffindbar gewesen und bei den oben genannten Untersuchungen aber sogar als „Fehler“ aufgefallen wäre, ist: In dem Text „Engel in der Nacht“ kommt das Wort „Engel“ versteckt in dem Wort „Treppengeländer“ vor. Hier wird ersichtlich, dass im Gegensatz zu statistisch, linguistischen Forschungsmethoden in der Literaturwissenschaft ein einziger Fund bedeutsam sein kann. Die digitalen Methoden haben sich dort am brauchbarsten erwiesen, wo ihr „,sinnhaftes“ Anwenden aufgehört, und das „Unsinnige“ begonnen hat.

Deshalb sollte immer bedacht werden, dass es bei allen Arbeiten mit computerunterstützten Methoden keine allgemeingültige Lösung gibt und die digitale Bearbeitung nur ein Werkzeug darstellt. Die Forschungsfragen und -aufgaben, müssen sich durch den Text ergeben und mit literaturwissenschaftlichen Fragestellungen bearbeitet werden - dies ist auch die Grundlage für corpusbasierte Literaturwissenschaft.

\section{Mapping :aichinger?}

Im Folgenden sollen drei kartierende Visualisierungsformen disktutiert werden. Das erste Ziel des Projekts :aichinger war es, alle im Werk genannten Orte zu erfassen. Da sich Aichingers Texte nicht in einer einfachen Weise auf die sogenannte 'Realwelt' beziehen, war diese Aufgabe zum Scheitern verurteilt. Die herkömmliche Vorstellung einer Landkarte stellt die realräumlichen Bezüge dar. Üblicherweise werden solche Relationen in räumlichen Maßstäben gemessen (Meter und seine Minder- bzw. Vervielfachungen). Vorstellbar wäre aber auch ein zeitlicher oder semantischer Maßstab. Selbst wenn die Distanzen in Metern gemessen werden, stellt sich aber die Frage, was dabei gemessen wird: die kürzeste Luftlinie, die unterschiedlichen Autostraßen-Verbindungen oder die Zug-Strecke? Abhängig davon, was gemessen wird, entstehen unterschiedliche Karten.

\subsection{Realkarten}

Die erste Karte, die hier diskutiert werden soll, verortet Ortsnamen auf einer gewöhnlichen, räumlichen „Landkarte“ (vgl. Fig. 1). Darin ist zunächst ein klarer Fokus auf die sogenannte westliche Kultursphäre 


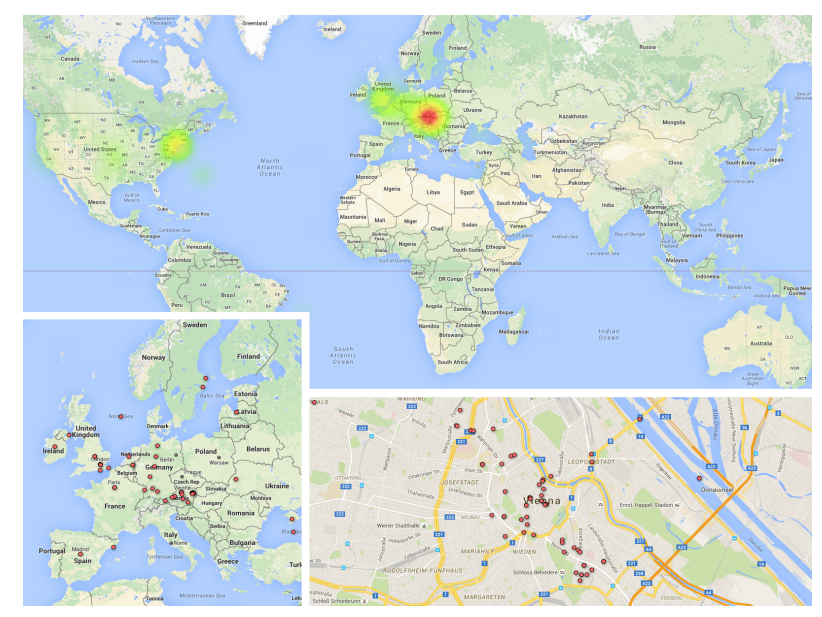

Abbildung 1: Verortung von Realort-Nennungen im Werk Ilse Aichingers.

(Zentraleuropa, USA) zu diagnostizieren. Am häufigsten werden Orte in Wien genannt; g efolgt von Orten in England - dem „Sehnsuchtsort” des Schreibens Aichingers (Ivanovic, 2011) - und Orten in den Vereinigten Staaten von Amerika.

In Wien selbst befinden sich die meisten Markierungen in Gegenden, in denen Ilse Aichinger gewohnt hat: das Fasanviertel im dritten Bezirk und die Gegend des ehemaligen Hauptquartiers der Gestapo, wo sie auch die sie prägende Deportation ihrer Großmutter erlebte. Ebenfalls häufig sind Orte in der Nähe ihres Wohnortes an der deutsch-österreichischen Grenze und im Salzkammergut, wo ihre Familie häufig Urlaub gemacht hat. Frankfurt, das damalige Verlagszentrum im deutschsprachigen Raum und kurzzeitiger Wohnort Aichingers als sie beim S. Fischer-Verlag als Lektorin gearbeitet hat, scheint nicht bedeutsam zu sein. - Eine biographische Landkarte, ließe sich also sagen.

Sieht man sich aber die Ortsnennungen genauer an, so stellt sich dieser Befund in einem etwas anderen Licht dar. Beispielsweise am Anfang der „größeren Hoffnung“. Dort heißt es, dass ,,[r]und um das Kap der Guten Hoffnung [...] das Meer dunkel“" werde oder dass ,eine Fliege [...] von Dover nach Calais“ krieche (Aichinger, 1991a, 9). Die genannten Ortsnamen beziehen sich dabei aber nicht auf die realen Orte, sondern auf die auf einer Landkarte verzeichnete Namen der Orte, wie erst aus den nächsten Sätzen klar wird.

Es sind also nicht in erster Linie reale Orte, von denen der Text hier spricht, sondern Ortsnamen. Ihre Bedeutungen erwachsen nicht nur aus deren bloßen Verortungen (wiewohl diese auch eine Rolle spielen können), sondern maßgeblich auch von ihren literarischen Funktionen. Die Landschaften, welche die Ortsnamen aufrufen, fungieren nicht als „Kulisse“ (Aichinger, 1991e, 179-180), sondern sind Teil einer literarischen Bedeutungs-Konstruktion, deren Bedeutung sich nicht in einer georeferenziellen Verortung erübrigt.

Das wird besonders deutlich, wenn „Die größere Hoffnung“ mit dem bereits erwähnten Satz beginnt, in dem sich das Meer rund um das „Kap der Guten Hoffnung“ verdunkelt. Oder auch in dem expliziten Spiel mit Namen, wie mit dem Ortsnamen „Aberdeen“, wenn es heißt, dass dieser „,mit dem ,Aber-“ am Beginn einem Widerstand ähnlich [sah]" (Aichinger, 2003 12 04). (Vgl. dazu auch Abschnitt 3 dieses Artikels.)

Peter Handke sagte in einem Interview über das Werk Ilse Aichingers: „Man spürt, dass die [Zauberformeln] aus einer starken, vorgezeichneten Realtität kommen. Diese Realität ist aber nicht ... nicht auf keiner Landkarte verzeichnet «1 Die Landkarte, von der Handke spricht und von der er die Texte Aichingers abzugrenzen bestrebt ist, fußt auf einem alltäglichen Verständnis von Karten, in dessen Denkrahmen ein direktes kausales Verhältnis zwischen Realität und ihrer Darstellung (auf einer Karte) angesetzt wird. Die realen innerweltlichen Relationen zeichneten sich, laut diesem Schema, wie auf einer Fotografie in der Darstellung ein. Dabei wird aber vernachlässigt, dass Darstellungen gestaltet, das heißt konstruiert sind: Bestimmte Aspekte müssen gekürzt, andere hinzugefügt werden - je nachdem, welchen Zwecken die

\footnotetext{
${ }^{1}$ Peter Handke in „Schreiben ist Sterben lernen...” (Film von Brita Steinwendtner, 1991, ORF-Archiv).
} 


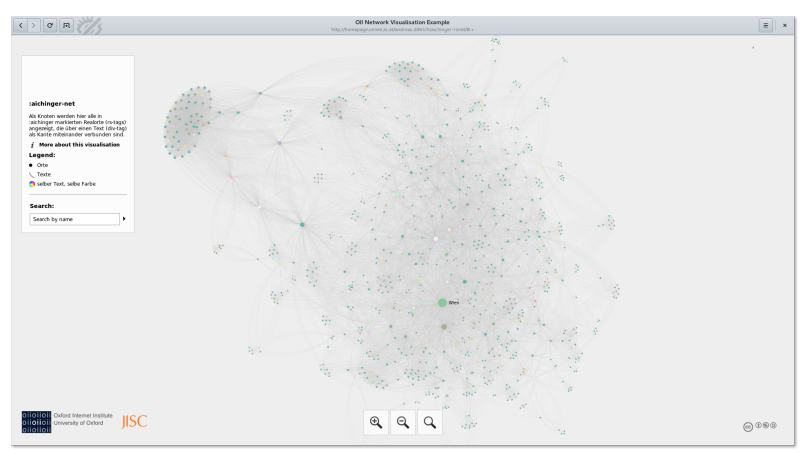

Abbildung 2: Die Karte der miteinander in Beziehung stehenden Ortsnamen. Online: http://www. aac.ac.at/oort/aichingernet/rsnet

jeweilige Karte zu dienen hat. Im Fall herkömmlicher Landkarten bemisst sich der Zweck an räumlichen Relationen, die mit bestimmten Werkzeugen und bestimmten Maßen vermessen werden können. Darauf ist unsere Welt eingestellt. Und so können solche Karten häufig auch nützlich sein. Auch lassen sich von ihnen Bedeutungen ablesen und Fragen bearbeiten, die sich auf das literarisch-bedeutungsvolle Verhältnis von Orten beziehen. Der Nutzen herkömmlicher Landkarten für literaturwissenschaftliche Untersuchungen konnte schon öfter aufgezeigt werden, beispielsweise von Moretti (1999).

\subsection{WOrt-Karten}

Es gibt aber auch andere Formen, Relationen von Namen resp. Ortsnamen räumlich darzustellen. Beispielsweise eine Form, die gewöhnlich Netzwerk, hier aber Karte genannt werden soll, da sie die Funktion einer Karte ausübt. Darin können Ortsnamen in einem abstrakten Raum verortet und miteinander in Beziehungen gesetzt werden, vgl. Fig. 2. Jeder Punkt (Knoten) auf dieser interaktiven Karte ist ein Ortsname und jede Linie, welche diese Punkte verbindet (Kante), ist eine Texteinheit. Auch hier ist ersichtlich, dass die Wortform „Wien” eine zentrale Stellung einnimmt. Sich bildende Ballungen (Cluster) bestehen aus Ortsnamen, die besonderst häufig in nur einem gemeinsamen Text vorkommen. Der große Cluster im Nordwesten der Karte versammelt Ortsnamen des Textes „Nachricht vom Tag” (Aichinger, 1991b, 146-156): Ein Text in dem von der Reise eines Tages durch viele verschiedene Orte bzw. Ortsnamen erzählt wird.

Werden umgekehrt die Texteinheiten als Knoten und die Ortsnennungen als Kanten dargestellt, wird ersichtlich, welche Texte die selben Ortsnamen nennen. Das gewährt einen intuitiven Überblick aller ortsnamenbezogenen Verbindungen zwischen den Texten. Der Text „Kleist, Moos, Fasane” (1987 im gleichnamigen Band erschienen) nennt beispielsweise viele Ortsnamen, die auch in den Texten nach 2000 genannt werden. Diesen Texten ist gemeinsam, dass sie einem ,autobiographischen Schreiben“ (Wagner-Egelhaaf) zuzuschreiben sind.

\subsection{Wort-Netzwerke}

In „schlechte Wörter“ von Aichinger heißt es, dass ,,[n]iemand [...] von mir verlangen [kann], daß ich Zusammenhänge herstelle, solange sie vermeidbar sind“" (Aichinger, 1991d, 12). Deswegen verwende sie zum Beispiel nicht mehr die Präposition „während“, welche einen zeitlichen Zusammenhang bedeuten würde, sondern die Konjunktion ,und“ in ihrer bloß aneinanderreihenden Funktion. Trotzdem, so könnte weiter gedacht werden, entstehen dabei aber Verbindungen zwischen den Wörtern. Diese können in Form eines Netzwerks zur Darstellung gebracht werden, in welchem nicht nur direkte, sondern auch indirekte Verbindungen ablesbar sind.

Dazu wurde die interaktive Arbeitsumgebung „RelFinder“ verwendet, welche für die Erkundung von Relationen in der Wikidata-Datenbank entwickelt worden ist (Heim et al., 2010) und durch seine Anknüpfbarkeit an andere RDF-Datenbanken auch für literaturwissenschaftliche Zwecke genutzt werden kann. Die vom TreeTagger erkannten Lemmata wurden in einen Triplestore geladen, an den der RelFinder über eine SPARQL-Schnittstelle angeschlossen ist. Gesucht werden können Lemmata, ganze Texteinheiten 


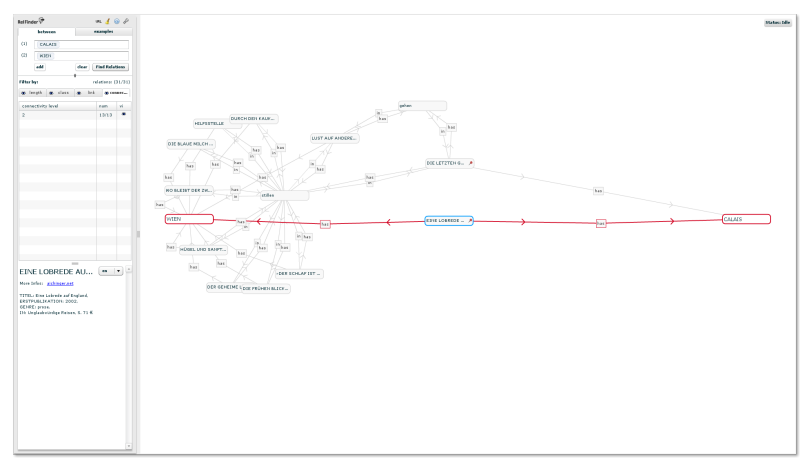

Abbildung 3: Wort-Relationen finden mit dem RelFinder.

und/oder die von der Arbeitsgruppe markierten Ortsnamen.

In Fig. 3 sind alle direkten und indirekten Verbindungen zwischen den Ortsnamen „Wien“ und „Calais“ dargestellt. Calais wird in nur einem Text gemeinsam mit Wien erwähnt (in „Eine Lobrede auf England"). Die anderen Verbindungen zwischen den beiden Ortsnamen sind vermittelt: In neun Texten wird „Wien“ mit „stillen” gebraucht - ein Wort, das nur in einem Text mit „Calais“ verwendet wird (in „Die letzten Gäste“, in: Aichinger (2005, 143-145)). In beiden Texten ist der Tod (einmal der eines engen Freundes und einmal der eigene) und das damit verbundene Begräbnis Thema: der stille Schläfer (Richard Reichensperger) mit seinem stillen Sarg und ein Seebegräbnis zwischen Dover und Calais.

\section{W-Ortspielereien mit A im Aichstand}

Die Grundlage der folgenden Untersuchungen bildet das Aichinger-Standard-Corpus (kurz: Aichstand), welches ein Subcorpus zum :aichinger Corpus darstellt und 179 Zeitungsartikel enthält. Zu diesen Artikeln von Aichinger, die zwischen 2000 und 2004 als Kolumne in der Tageszeitung Der Standard publiziert wurden, zählen das „Journal des Verschwindens“(51 Artikel), die „Unglaubwürdigen Reisen“ (85) und die „Schattenspiele“ (43). Zu jeder Kolumne wurden drei Korpora generiert, da jeder Artikel dreimal veröffentlicht wurde: als Print, Online-Publikation und in Buchform.

Ilse Aichingers Kolumnen-Artikel unterscheiden sich von den anderen Texten im Gesamtwerk, da sie in Serie veröffentlicht wurden. Die Autorin hat für gewöhnlich wöchentlich handschriftlich einen neuen Text verfasst, diesen am Donnerstag in der Standard-Redaktion für die Kolumne abgegeben, die jeden Freitag im Kulturteil erschien. Jeweils zwei Jahre später folgten zwei Bände, welche eine Auswahl dieser Texte versammelte Film und Verhängnis (2001) und Unglaubwürdige Reisen (2005).

$\mathrm{Zu}$ jedem Artikel existieren verschiedene Textvarianten: die Manuskripte, die Printartikel in der Tageszeitung, die jeweilige Onlineversion auf www.derstandard.at und die Texte im jeweiligen Sammelband.

Mit der Möglichkeit, digitale Korpora nebeneinander zu sehen, entstehen neue Möglichkeiten von W-Ortspielereien:

Betrachtet man den Artikel „Das Aberdeenspiel“ (2004) aus der Kolumne „Schattenspiele“, ausgehend von der Buchfassung, in der das entsprechende Manuskript und der Text nebeneinandergelegt wurden (Aichinger, 2005, 116-117), fällt auf, dass der Lesetext stark vom Manuskript-Text abweicht. Am Ende des Lesetexts (im Buch) wurde das Datum der Veröffentlichung im Standard angegeben.

Bei der Erarbeitung des Spätwerks stellte sich heraus, dass zum „Aberdeenspiel“ zwei Texte existieren, sowohl der Artikel mit dem Titel „Das Aberdeenspiel“ (Aichinger, 200407 02), erstmals am 02.07.2004 im Standard publiziert, als auch „Die letzten Schritte in Aberdeen“ vom 04.12.2003 (Aichinger, 200312 04).

Beide Artikel handeln vom Aberdeenspiel: Ein Einschlafspiel, das Ilse Aichinger, so erinnert sie sich, als Kind gelegentlich spielte. Im Manuskript steht: „Ein sehr frühes Spiel hieß das Aberdeenspiel. Es grenzte an diese noch älteren Spiele. Flüsse, Farben, Blumen, Vögel. Man blieb für sich dabei und konnte es über Aachen, Artstetten und Albern hinspielen [... ]." Beim Aberdeenspiel werden in alphabetischer 
Reihenfolge solange Orte aufgezählt bis einem keine mehr zu dem jeweiligen Buchstaben einfallen. Dann wird das Spiel mit dem nächsten Buchstaben fortgesetzt.

Um herauszufinden, wie die Textversionen vom Aberdeenspiel zueinander in Beziehung stehen, wurden mit Hilfe eines Vergleichstools die Texte nebeneinandergelegt, um Gemeinsamkeiten und Unterschiede zu prüfen. So konnte herausgefunden werden, dass ausgehend von einem der „fliegenden, fliehenden“ Manuskriptblätter (Fässler, 2005, 8), sowohl Textstellen in dem Artikel „Die letzten Schritte in Aberdeen“ zu finden sind, andere wiederum im Artikel „Das Aberdeenspiel“.

Mit Hilfe des digitalen Corpus :aichinger lässt sich nun das von Aichinger in den Raum gestellte Spiel mit ihrem spezifischen Vokabular durchspielen und alle im Corpus markierten Ortsnamen mit dem Anfangsbuchstaben A ausgeben, was rund 40 unikale Treffer ergibt und in einer Auswahl hier zum Abschluss abgedruckt werden soll: Aachen, Aalen, Aberdeen, Abilene, Afrika, AKH, Alabama, Alaska, Albany, Albern, Albertina, Alsergrund, Alt-Aussee, Amalfi, Amberg, America, Amerika, Amsterdam, Anatolien, Andernach, Ansbach, Arizona, Arosa, Arsenal, Asien, Aspangbahnhof, Athen, Atlanta, Atlantik, Attersee, Auckland, Augarten, Auschwitz, Australien, Austria, Auvergne.

\section{References}

Ilse Aichinger. 1991a. Werke. Die größere Hoffnung. S. Fischer Verlag.

Ilse Aichinger. 1991b. Werke. Eliza Eliza. S. Fischer Verlag.

Ilse Aichinger. 1991c. Werke. Kleist, Moos, Fasane. S. Fischer Verlag.

Ilse Aichinger. 1991d. Werke. Schlechte Wörter. S. Fischer Verlag.

Ilse Aichinger. 1991e. Werke. Zu keiner Stunde. S. Fischer Verlag.

Ilse Aichinger. 2003-12-04. Die letzten Schritte in Aberdeen. Der Standard.

Ilse Aichinger. 2004-07-02. Das aberdeenspiel. Der Standard, page 26.

Ilse Aichinger. 2005. Unglaubwürdige Reisen. S. Fischer Verlag.

Ilse Aichinger. 2006. Subtexte. Edition Korrespondenzen.

Ilse Aichinger. 2011. Es muss gar nichts bleiben. Edition Korrespondenzen, 2 edition.

Simone Fässler. 2005. Vorwort. In Unglaubwürdige Reisen, pages 7-12. S. Fischer Verlag.

Simone Fässler. 2011. Von Wien her, auf Wien hin. Böhlau.

Philipp Heim, Steffen Lohmann, and Timo Stegemann. 2010. Interactive Relationship Discovery via the Semantic Web. In Proceedings of the 7th Extended Semantic Web Conference (ESWC 2010), volume 6088 of LNCS, pages 303-317. Springer.

Christine Ivanovic. 2011. Nach England! Zur Geschichte einer Sehnsucht. In Rüdiger Görner, Christine Ivanovic, and Sugi Shindo, editors, Wort-Anker Werfen, pages 87-101. Königshausen \& Neumann.

Franco Moretti. 1999. Atlas des europäischen Romans. DuMont.

Helmut Schmid. 1995. Improvements in Part-of-Speech Tagging with an Application to German. In Proceedings of the ACL SIGDAT-Workshop.

Martina Wagner-Egelhaaf. 2005. Autobiographie. Verlag J.B. Metzler, 2 edition. 